\title{
Spontaneous Splenic Haematoma Presenting as Perforated Acute Appendicitis in Young Adult
}

\author{
Mohd Shafiq Rahman ${ }^{1}$, Norly Salleh ${ }^{1,2}$ \\ ${ }^{1}$ Department of Surgery, Hospital Segamat, KM 6, Jalan Genuang, 85000 Segamat, Johor, Malaysia. \\ ${ }^{2}$ Satellite Clinical Research Centre, Hospital Segamat, KM 6, Jalan Genuang, 85000 Segamat, Johor, Malaysia \\ DOI: 10.29322/IJSRP.10.11.2020.p10767 \\ http://dx.doi.org/10.29322/IJSRP.10.11.2020.p10767
}

\begin{abstract}
Background: Spontaneous splenic haematoma, in the absence of trauma is rare in young immunocompetent adult. Malignancy, infection, and medication are some of the aetiologies identified. Diagnosing spontaneous splenic haematoma is a challenge as it may mimic other disease. Case Presentation: A 21-year-old male presented to emergency district hospital with umbilical pain migrating to right iliac fossa. Physical examination revealed right iliac fossa tenderness but no peritonism. Provisional diagnosis of perforated appendicitis was made and patient was planned for open appendicectomy via Lanz incision. Reassessment revealed generalized abdominal pain and patient underwent lower midline laparotomy. Intraoperatively there was $1.8 \mathrm{~L}$ of haemoperitoneum and haematoma was found at splenic hilum. Splenectomy was performed and patient was discharged well with prophylaxis antibiotic. Conclusion: Spontaneous splenic haematoma is rare and should be considered in young immunocompetent adult presenting with abdominal pain.
\end{abstract}

Index Terms- spleen, spontaneous, haematoma, atraumatic, rupture

\section{INTRODUCTION}

$\mathrm{S}^{\mathrm{n}}$ pontaneous splenic haematoma is rare and potentially lifethreatening. The incidence is between $0.1 \%$ to $0.5 \%$. A systemic review of atraumatic splenic rupture by Renzulli et al found that 845 cases was reported from 1980 to $2008^{1}$. Most reported cases of spontaneous splenic haematoma have background history of chronic medical illness that may predispose to this condition, making it extremely rare in young, immunocompetent adult. We present a case of spontaneous splenic haematoma in young adult with no prior medical condition with atypical presentation mimicking as perforated appendicitis.

\section{CASE REPORT}

21-year-old male with no prior medical illness presented to emergency department with abdominal pain of one-day duration. The pain started at umbilical area and later migrated to right iliac fossa. He denied any fever, nausea or vomiting. No history of trauma was reported. Upon initial assessment, patient was not tachypnoeic or tachycardic. Initial blood pressure was 80/45 $\mathrm{mmHg}$, but it rose to $109 / 51 \mathrm{mmHg}$ after fluid resuscitation.
Physical examination revealed local guarding and tenderness over right iliac fossa. His haemoglobin level was normal at 11.7 $\mathrm{g} / \mathrm{dl}$, white cell count $\left(17.1 \times 10^{3} / \mathrm{uL}\right)$, urea $(4.4 \mathrm{mmol} / \mathrm{L})$ and creatitine $(141 \mathrm{mmol} / \mathrm{L})$. The rest of his blood parameters were within normal range. Electrocardiogram and chest radiography were also done and they turned out to be normal.

Provisional diagnosis of perforated appendicitis was made. Open appendicectomy via Lanz incision was arranged. However, operation was changed to lower midline laparotomy as patient developed generalized abdominal guarding and tenderness upon reassessment one hour later.

Intra-operatively $1.8 \mathrm{~L}$ of blood was found in the peritoneum. His appendix was normal. There was haematoma at the splenic hilum and tail of the pancreas. Abdominal packing was done and urgent contrast enhanced $\mathrm{CT}$ angiography was arranged. The scan revealed minimal haematoma at region of splenic hilum with no evidence of intra-abdominal active bleeder or abnormal vascular dilatation (Figure 1). Pancreas was homogenously enhanced with no peripancreatic fluid or collection. Relaparotomy, abdominal packing removal and splenectomy was performed 24 hours after first operation. Histopathology of the removed spleen showed congested spleen with haematoma.

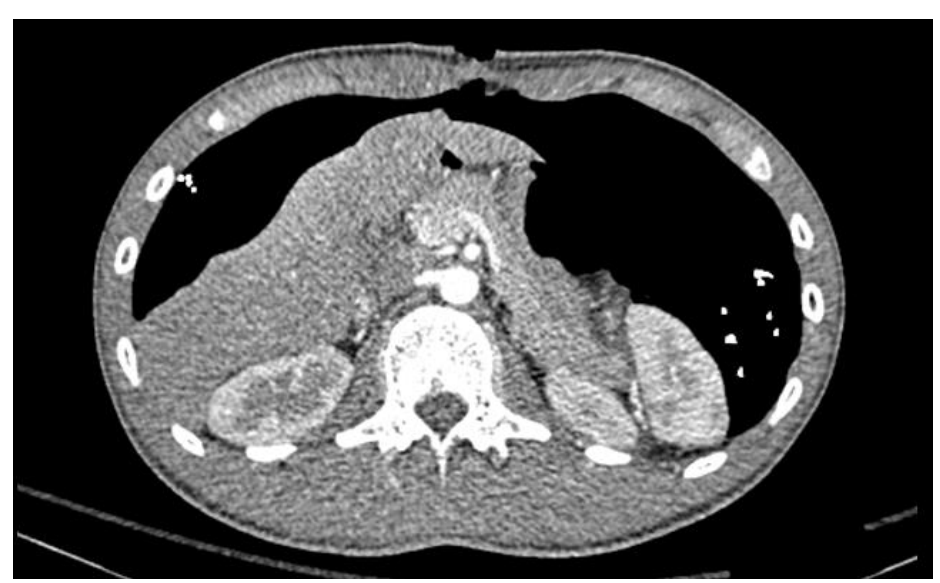

Figure 1: Haematoma at splenic hilum with no evidence of intraabdominal bleeder or abnormal vascular dilatation. 
Post-operatively, patient's condition remained stable. He was given post splenectomy vaccination and prophylaxis antibiotic upon discharge as per local guideline. He was discharged well on day six post operation.

\section{DISCUSSION}

Malignancy, infection, inflammation, drug and treatment-related are some of the aetiology identified for spontaneous splenic haemtaoma[1]. Hepatitis, malaria, Epstein-Barr virus infection and cytomegalovirus infection are some of the infective causes [2]. Lianos et al listed five cases of cytomegalovirus related splenic haematoma. All patients were between the age of 22 to 30-year-old with three of them has no comorbid (immunocompetent adult) [3]. Cytomegalovirus infection could be the underlying aetiology in our case too. However, we were unable to do any confirmatory test due to resource constraint.

The mechanism behind the spontaneous splenic haematoma is still not fully understood. One of the mechanisms implicated is that cellular hyperplasia and engorgement resulting in increased intrasplenic tension [3][4]. Other mechanism involves reticule endothelial hyperplasia resulting in vascular occlusion leading to thrombosis and infarction ${ }^{3,4}$.

Patient with spontaneous splenic haematoma commonly presents with non-specific upper quadrant abdominal pain. Blood investigations may reveal the underlying aetiology but it can be normal too. In our case, patient presented with migratory abdominal pain that was very typical of appendicitis, which may contribute to our decision to proceed with operation without any prior imaging.

Two modalities use for diagnosing spontaneous splenic haematoma are ultrasound and computer topography (CT). Depending on the haemodynamic status of the patient, exploratory laparotomy may be performed prior to any imaging and diagnosis is made intraoperatively [5][6]. Conservative management with blood transfusion and correction of underlying aetiology can be taken in haemodynamically stable patient [5][6].

\section{CONCLUSION}

In conclusion, spontaneous splenic haematoma is rare especially in young adult with no prior medical illness. Awareness of this condition is important in order for the diagnosis to be made and managed accordingly.

\section{APPENDIX}

Appendixes, if needed, appear before the acknowledgment.

\section{ACKNOWLEDGMENT}

None

\section{REFERENCES}

[1] Renzulli, P., Hostettler, A., Schoepfer, A.M., Gloor, B. and Candinas, D. (2009), Systematic review of atraumatic splenic rupture. Br J Surg, 96: 11141121. doi:10.1002/bjs.6737

[2] Barrak D, Ramly EP, Chouillard E, Khoury M. Chronic spontaneous idiopathic spleen hematoma presenting as a large cystic tumor: a case report with review of the literature. J Surg Case Rep. 2014;2014(6): rju060. Published 2014 Jun 10. doi:10.1093/jscr/rju060

[3] Lianos G, Ignatiadou E, Bali C, Harissis H, Katsios C. Successful Nonoperative Management of Spontaneous Splenic Hematoma and Hemoperitoneum due to CMV Infection. Case Rep Gastrointest Med. 2012; 2012:328474. doi:10.1155/2012/328474

[4] Y. Yagmur, I. H. Kara, M. Aldemir, H. Buyukbayram, I. H.Tacyildiz, and C. Keles, "Spontaneous rupture of malarial spleen: two case reports and review of literature," Critical Care, vol. 4, no. 5, pp. 309-313, 2000

[5] Pandiaraja J. Spontaneous Splenic Haematoma in a Patient on Hemodialysis A Case Report. Indian J Nephrol. 2017;27(6):475-477. doi: 10.4103/ijn.IJN_285_16

[6] Kocael PC, Simsek O, Bilgin IA, et al. Characteristics of patients with spontaneous splenic rupture. Int Surg. 2014;99(6):714-718. doi:10.9738/INTSURG-D-14-00143.1

\section{AUTHORS}

First Author - Mohd Shafiq Rahman, MBBS, Department of Surgery, Hospital Segamat, Johor, Malaysia

Second Author - Norly Salleh, MS Gen Surg, Department of Surgery, sCRC Hospital Segamat, Johor, Malaysia

Correspondence Author - Mohd Shafiq Rahman,

mr.shafiq1689@gmail.com,6079433333 